\title{
Design and simulation of a combined serpentine T-shape magnetorheological brake
}

\author{
Faishal Harish Hidayatullah ${ }^{1}$, Ubaidillah ${ }^{2}$, Endra Dwi Purnomo ${ }^{3}$, \\ Dominicus Danardono Dwi Prija Tjahjana ${ }^{4}$, Ilham Bagus Wiranto ${ }^{5}$ \\ $1,2,3,4,5$ Mechanical Engineering, Universitas Sebelas Maret, Surakarta, Indonesia \\ ${ }^{2}$ National Center for Sustainable Transportation Technology, Bandung, Indonesia
}

\section{Article Info}

Article history:

Received Sep 15, 2018

Revised Nov 21, 2018

Accepted Dec 22018

\section{Keywords:}

FEMM

Magnetorheological brake

Magnetorheological fluid

\begin{abstract}
A magnetorheological brake (MRB) is a device to dissipate rotational energy using magnetorheological fluids (MRF). MRB can change its braking torque quickly in response to external magnetic field strength. The brake is rotational, utilizing the MRF in shear mode. In this study, the geometrical design of the MRB, magnetic circuit and MRF flow path is addressed. Mathematical models are presented that describe the braking torque of the MRB. A novel prototype is introduced combining T-shape rotor model with serpentine flux magnetic circuit configuration. The rotor member is selected to direct the flux concentration at that location. Serpentine flux configuration is selected to achieve higher torque without increasing the size of MRB by activated more surface area of MRF with the magnetic flux. The finite element method is used to evaluate the magnetic flux density in MRB using FEMM 4.2. FEMM results showed that this novel design could provide sufficient magnetic flux along MRF flow path. Finally, the influence of input current to the MRB on braking torque is investigated. It is found that the braking torque in MRB increases with the increase of the input current. The prototype is formulated as foot-drop prevention orthotic. The MRB would be further integrated into ankle-foot orthoses for post-stroke patients. The design is formulated as a preliminary geometrical design, aiming to obtain the minimum required braking torque.
\end{abstract}

Copyright $\odot 2019$ Institute of Advanced Engineering and Science. All rights reserved.

\section{Corresponding Author:}

Ubaidillah,

Department of Mechanical Engineering,

Sebelas Maret University,

Jl. Ir. Sutami 36A, Kentingan, Surakarta, Indonesia.

Email: ubaidillah_ft@staff.uns.ac.id

\section{INTRODUCTION}

Magnetorheological Fluid (MRF) is a fluid that is responsive to magnetic fields and is categorized as smart fluid or controllable fluid [1]-[2]. MRF consists of carbonyl iron particle magnetized to micron size suspended in non-magnetic fluids. Carbonyl iron particle has a spherical particle and has a high magnetic field absorption; carbonyl iron particle used to formulate MRF. In the absence of magnetic field MRF will remain in free-flowing fluid conditions, but under the influence of magnetic field, the viscosity of MRF will increase in less than $10 \mathrm{~ms}[3]-[5]$.

Since the first time introduced by Rabinow on "The Magnetic Fluid Clutch" in 1948 development of MR-based device has been drastically increased [1]. The ability of MRF to increase viscosity when affected by magnetic field causes MRF has been widely applied as vibration damping device. MRF is also applied as a seismic insulator in the construction of buildings to dampen the vibrations generated by the earthquake [6]. MRF in the automotive industry has been presented as a magnetorheological damper (MR Damper) to 
dampen vibration caused by the road surface. MR Damper damping performance may be dynamically varied to provide stability across different road conditions [7]-[9].

Magnetorheological Brake (MRB) is one application of MRF. In the automotive industry, MRB has advantages when compared with conventional brakes including instant response time, does not require replacement brake pad, and compact dimensions [10]. So far, the application for high torque MR brake was conducted by Hung et al. utilizing the T-Shape MR brake in middle size motorcycle. The maximum torque achieved by the optimized design was $441 \mathrm{Nm}$ at cruising speed $120 \mathrm{~km} / \mathrm{h}$ [11]. This excellent result was achieved after optimization of the MR brake design.

Moreover, the type of MRB is divided into several types including disks, drums, multiple disks and T-shape brakes [12]. The construction of MRB consists of rotor, stator, and coil. Commonly, to achieve higher braking torque researchers optimize the performance of MRB by developing rotor model, stator configuration, and coil configuration [13],[14]. Ubaidillah et al. [13] have presented different stator configurations between magnetic and non-magnetic materials called serpentine flux to distribute flux density evenly along the MRF flow path. Shiao et al. [14] were presented multiple pole coil configuration to strengthen flux density along the MRF gap.

Since, the reliable performance and compact dimension of MRB researcher has been developed MRB as a rehabilitation device. An MRB prosthetic knee, called Rheo Knee manufactured by the company Ossur Inc. [15] MRB stiffness may be varied in real-time as the amputee walks. Ubaidillah et al. [13] prototyped an MRB as foot-drop prevention orthotic device for a post-stroke patient. Avraam et al. [16] proposed MRB as wrist rehabilitation device for muscular exercise. Gudmundsson et al. [17] also prototyped an MRB used a novel perfluorinated polyether (PFPE)-based MRF is introduced, whose properties are tailored for the prosthetic knee.

Therefore, this article proposed a novel design of MRB combining T-shape model MRB and serpentine flux stator configuration to achieve the minimum required braking torque that is about $1.5 \mathrm{Nm}$ in a compact size. The prototype would be integrated as foot-drop prevention device to support the patient anklefoot. Since the prototype would be placed in an ankle, thus the mass and dimensions of the prototype should be considered as light as possible. The discussion covers design, working principle, mathematical model, magnetic simulation and braking torque prediction.

\section{DESIGN OF MAGNETORHEOLOGICAL}

For this specific novel MRB prototype, there is two concept adapted in this study. The first concept was MRB T-shape model firstly introduced by Carlson et al. [18] T-shaped construction is advantageously utilized to concentrate the effect of the MRF to the applied field of the T-shaped plate portion. Also the rotor member is selected to direct the flux concentration at that location. The second concept was serpentine flux MRB stator configuration conducted by Senkal et al. [19] to achieved higher torque without increasing the size of MRB by activated more surface area of MRF with the magnetic flux. Strategically, placing magnetically conductive with a ring of non-magnetically conductive material it is possible to bend the magnetic field and activated MRF along gap multiple times. Serpentine flux led to more compact brake design and enabled us to increase the braking torque.

For this specific novel MRB prototype, there is two concept adapted in this study. The first concept was MRB T-shape model firstly introduced by Carlson et al. [18] T-shaped construction is advantageously utilized to concentrate the effect of the MRF to the applied field of the T-shaped plate portion. Also the rotor member is selected to direct the flux concentration at that location. The second concept was serpentine flux MRB stator configuration conducted by Senkal et al. [19] to achieved higher torque without increasing the size of MRB by activated more surface area of MRF with the magnetic flux. Strategically, placing magnetically conductive with a ring of non-magnetically conductive material it is possible to bend the magnetic field and activated MRF along gap multiple times. Serpentine flux led to more compact brake design and enabled us to increase the braking torque.

The prototype of this specific MRB would be integrated into ankle-foot orthoses. Therefore, the size was limited to maximum constraint $60 \mathrm{~mm}$ of diameter and $30 \mathrm{~mm}$ of thickness. As shown, in Figure 1 the rotor is using T-shape model rotor and serpentine flux stator configuration. Stator configuration is a magnetic and non-magnetic material with a length of $8.5 \mathrm{~mm}$ and $3 \mathrm{~mm}$ respectively. MRF-132DG is utilized as a braking medium, MRF working in shear mode and flowing in $0.5 \mathrm{~mm}$ of the gap. For more details, the properties of MRF-132DG and list of parts for proposed MRB is shown in Tables 1 and 2, respectively. 


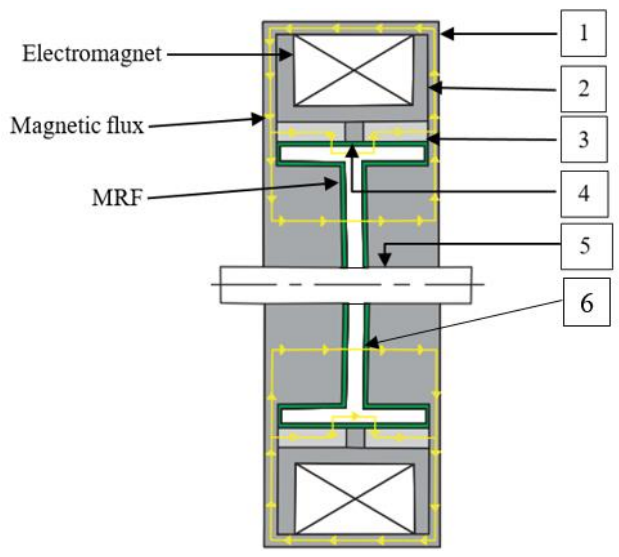

Figure 1. Schematic of MRB prototype

Table 1. Basic specification MRF-132DG

\begin{tabular}{cc}
\hline Property & Value \\
\hline Appearance & Dark gray liquid \\
Viscosity, Pa-s & 0.112 \\
Density, $\mathrm{g} / \mathrm{cm}^{3}$ & $2.95-3.15$ \\
Solid content by weight, $\%$ & 80.98 \\
Flashpoint, ${ }^{\circ} \mathrm{C}$ & $>150$ \\
Operating Temp., ${ }^{\circ} \mathrm{C}$ & -40 to +130 \\
\hline
\end{tabular}

Table 2. The list of parts for proposed MRB

\begin{tabular}{cccc}
\hline Part No. & Part & Type & Material \\
\hline 1 & Casing & Magnetic & Steel 1010 \\
2 & Bobbin & Non-Magnetic & Aluminium 1100 \\
3 & Stator Magnetic & Magnetic & Steel 1010 \\
4 & Stator Non-Magnetic & Non-Magnetic & Aluminium 1100 \\
5 & Shaft & Non-Magnetic & Aluminium 1100 \\
6 & T-shape Rotor & Magnetic & Steel 1010 \\
\hline
\end{tabular}

For T-shape brake, the basic equation is expressed in Equation 1:

$$
\tau=\tau_{y}(B)+\eta(\dot{\gamma}) \frac{R \dot{\theta}}{g}
$$

where, $\tau$ is shear stress, $\tau_{y}$ is shear stress field-dependent, $\dot{\gamma}$ is shear rate, $\dot{\theta}$ is angular velocity of rotor, $\mathrm{R}$ is radius of rotor, and g is MRF gap. Moreover, for torque calculation, there are 4 components to calculate the total braking torque transmitted $T_{b}$, including $T_{r 1}, T_{r 2}, T_{a 1}$, and $T_{a 2}$ are respectively, braking torque at T-leg radial face, $\mathrm{T}$-flange radial face, inner and outer annular face of $\mathrm{T}$-flange. Total torque transmitted represented as,

$$
T_{b}=2\left(T_{r 1}+T_{r 2}+T_{a 1}\right)+T_{a 2}
$$

Finally, $T_{r 1}, T_{r 2}, T_{a 1}, T_{a 2}$, and $T_{\eta}$ are described below, respectively.

$$
\begin{aligned}
& T_{r 1}=2 \pi \tau_{y} R_{i f}^{3} \\
& T_{r 2}=2 \pi \tau_{y}\left(R_{o f}^{3}-R_{i f}^{3}\right) \\
& T_{a 1}=2 \pi \tau_{y} R_{i f}^{3} L_{1} \\
& T_{a 2}=2 \pi \tau_{y} R_{o f}^{3} L_{2}
\end{aligned}
$$




\section{MAGNETIC CIRCUIT ANALYSIS}

It is essential to establish the effectiveness of the designed MRB was approved by magnetic circuit analysis. Magnetic circuit analysis will explain magnetic flux along the circuit, and it also could be relevant for magnetic flux density prediction generated by the circuit. According to Figure 2, there are five reluctances such as $R_{\text {coil }}, R_{\text {wall }}, R_{\text {rotor }}, R_{\text {stator steel }}, R_{M R F}$.

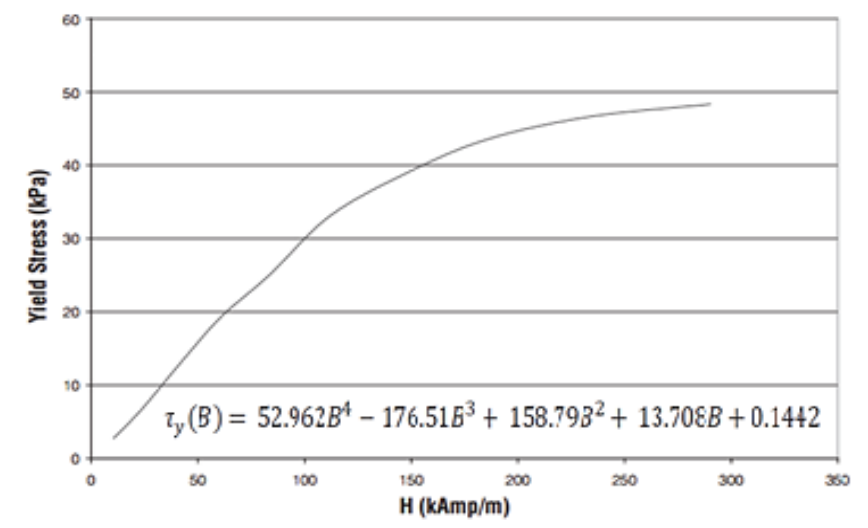

Figure 2. Relation of shear yield stress and magnetic field intensity of MRF-132DG

The reluctance of each section can be determined by Equation 7 as follows,

$$
R=\frac{L}{\mu A}
$$

where, $\mathrm{L}$ is distance passed by the magnetic flux in a single area, $\mu$ is the magnetic permeability, and $\mathrm{A}$ is the active area of the magnetic flux line. Based on the circuit, the total can be the summation of all reluctances stated in Equation 8.

$$
\begin{aligned}
& \Sigma R=R_{\text {coil }}+R_{\text {wall }}+R_{M R F}+R_{\text {rotor }}+R_{M R F}+R_{\text {stator steel }}+R_{M R F}+R_{\text {rotor }}+R_{M R F}+ \\
& R_{\text {stator steel }}+R_{M R F}
\end{aligned}
$$

The total electromotive force as expressed in Equation 9 below,

$$
\Sigma F=\phi \Sigma \mathrm{R}=\mathrm{NI}
$$

where, $\phi, \mathrm{N}$, and I are magnetic flux, wire turns in each coil, and the current passing through the coils, respectively.

\section{MAGNETIC FIELD ANALYSIS}

In this study, magnetic field analysis is really important to investigate magnetic flux density and magnetic flux path of the prototype using magnetic simulation software, FEMM (Finite Element Methods Magnetics) V 4.2. Magnetic field analysis used to evaluate the concentration of magnetic flux and considered the effective area of proposed MRB. MRF used in this device is MRF-132DG, MRF was flowing inside $0.5 \mathrm{~mm}$ of the gap. MRB was described in software simulation, along with rotor, bobbin, stator, and coil wire. All magnetic properties of MRB components is assigned as same as described in the design section. In Figure 3 presented MRB in a 2D axisymmetric meshed model of MRB in FEMM with matching material properties.

Figure 4 presented the simulated magnetic field distribution for the full magnetic circuit. The important notes are the magnetic flux that can influences the MRF properties is flux that passes through the gaps. Based on Figure 4, it can examine that flux path has passed through the casing, rotor, and stator. It is why the rheological properties of MRF only change when flux through this components. 


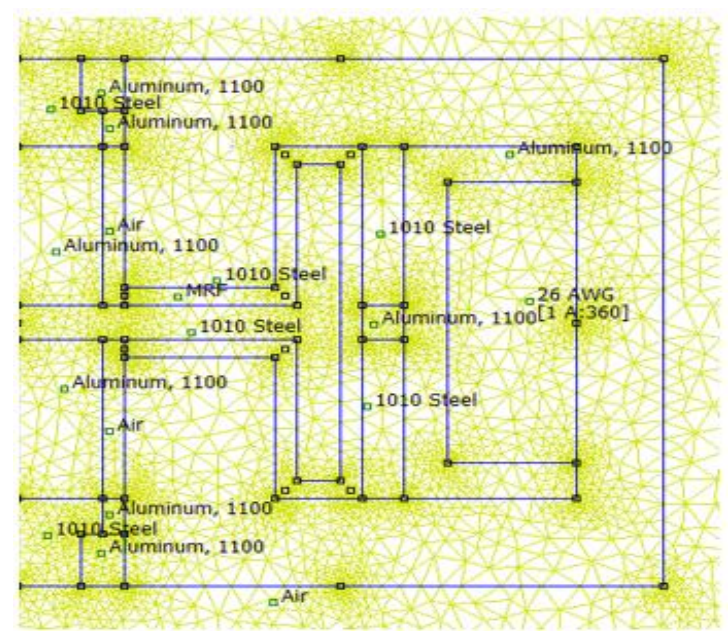

Figure 3. 2D axisymmetric meshed model of MRB

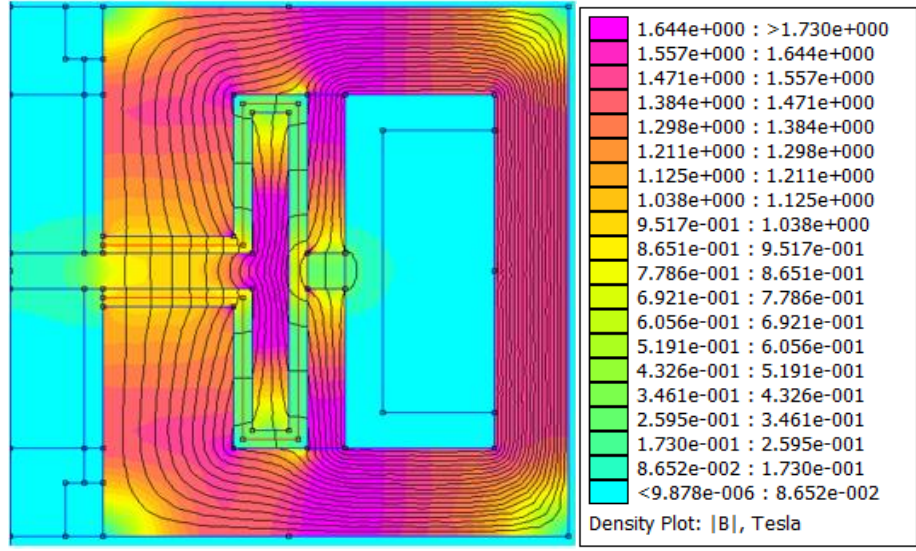

Figure 4. flux density distribution of MRB

From the results of magnetic simulation the material configuration of the shaft was concentrated the magnetic flux inside the channel shown in Figure 4. Therefore, in Figure 5 the magnetic flux density in the horizontal rotor area had the highest value of $0.86 \mathrm{~T}$. Meanwhile, the vertical rotor area has the lowest magnetic flux density of $0.25 \mathrm{~T}$ because the magnetic field was not concentrated in that area. Moreover, the serpentine flux stator configuration also successfully increased the magnetic flux density in the stator area by $0.52 \mathrm{~T}$.

1

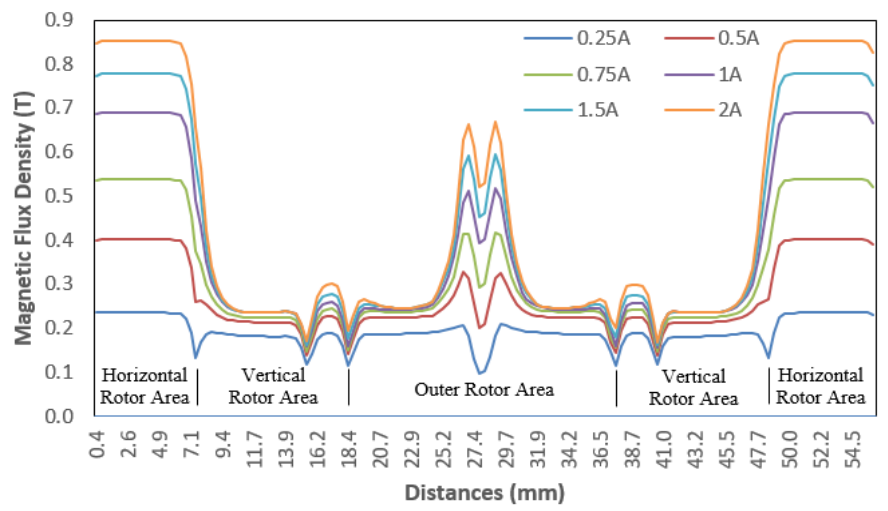

Figure 5. Relation of magnetic flux density along the gap and current 


\section{PERFORMANCE PREDICTION}

Figure 6 presented braking torque prediction of proposed MRB. Braking torque prediction was calculated from Equation 2, $T_{b}$ is the summation of the 4 component of torque $T_{r 1}, T_{r 2}, T_{a 1}$, and $T_{a 2}$. The first step to calculate braking torque were started by establish the magnetic flux data from magnetic simulation in FEMM. The off-state torque, $T_{\eta}$ could simply calculated from angular speed and MRF base viscosity.

The performance achieved by proposed MRB was satisfying. Since the proposed MRB had limited dimension. In Figure 6, evaluating the braking torque is significantly improve as the increase of applied current. The maximum torque achieved by proposed MRB is 2.1 N.m at 2 Ampere of current, and the offstate torque is 0.001 N.m. Since braking torque of MRB is dependent on the magnetic field, the applied current has an important role in increasing the braking torque. As the magnetic field strength increases, the braking torque of MRB can be significantly increased.

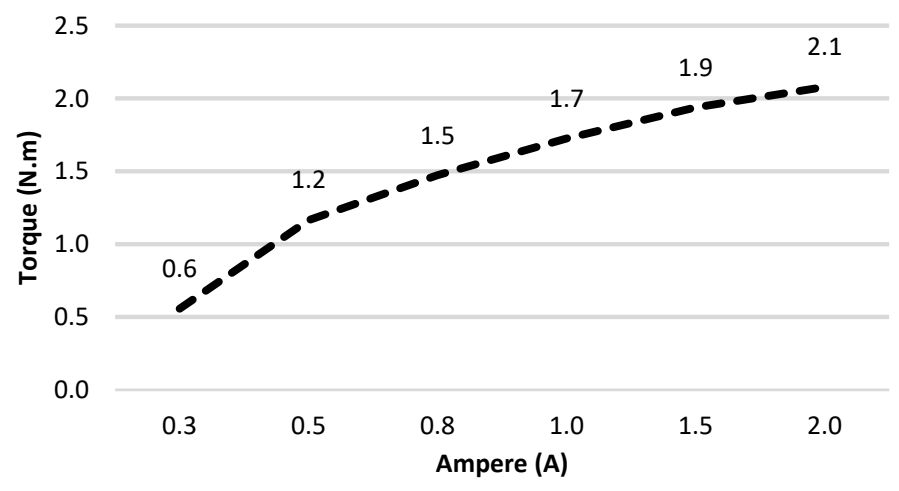

Figure 6. Relation of braking torque and current

\section{CONCLUSION}

Performance investigation of serpentine T-shape MRB has been sucessfully presented. According to the magnetostatic simulation, the serpentine flux stator configuration has been successfully bending the magnetic flux path in the MRF gap. Consequently, the active area of MRF was increasing. The maximum achieved braking torque is 2.1 N.m at 2 Ampere of current. Since braking torque of MRB is dependent on the magnetic field, the applied current has an important role in increasing the braking torque. As the magnetic field strength increases, the braking torque of MRB can be significantly increased. In the future, serpentine T-shape MRB design can be further developed to achieve higher braking torque.

\section{ACKNOWLEDGEMENTS}

Authors acknowledge UNS through Hibah Kolaborasi Internasional 2019 as well as USAID for partial support through SHERA Program-Centre for collaborative (CCR) National Center for Sustainable Transportation Technology (NCSTT) with contract No. IIE00000078-ITB-1.

\section{REFERENCES}

[1] J. Rabinow, "The Magnetic Fluid Clutch," Trans. Am. Inst. Electr. Eng., vol/issue: 67(2), pp. 1308-1315, 1948.

[2] P. Gadekar, et al., "Magnetorheological Fluid and its Applications," Int. J. Curr. Eng. Technol. IJCET INPRESSO Special Issue, vol/issue: 7(7), pp. 2277-4106, 2017.

[3] Ubaidillah, et al., "A new class of magnetorheological elastomers based on waste tire rubber and the characterization of their properties," Smart Mater. Struct., vol/issue: 25(11), pp. 1-15, 2016.

[4] U. Ubaidillah, et al., "Response of A Magnetorheologi cal Brake under Inertial Loads," Int. J. Electr. Eng. Informatics, vol/issue: 7(2), pp. 308-322, 2015.

[5] S. A. A. Aziz, et al., "Effects of multiwall carbon nanotubes on viscoelastic properties of magnetorheological elastomers," Smart Mater. Struct., vol/issue: 25(7), pp. 1-10, 2016.

[6] S. J. Dyke, et al., "Seismic response reduction using magnetorheological dampers," Proc. IFAC World Congr., pp. $145-150,1996$.

[7] F. Imaduddin, et al., "A design and modelling review of rotary magnetorheological damper," Mater. Des., vol. 51, pp. 575-591, 2013. 
[8] Y. B. Kazakov, et al., "Development of models of the magnetorheological fluid damper," J. Magn. Magn. Mater., vol. 431, pp. 269-272, 2017.

[9] J. De Jesus Lozoya-Santos, et al., "Control strategies for an automotive suspension with a MR damper," IFAC Proc., vol/issue: 18(1), pp. 1820-1825, 2011.

[10] K. Karakoc, et al., "Design considerations for an automotive magnetorheological brake," Mechatronics, vol/issue: 18(8), pp. 434-447, 2008.

[11] N. Q. Hung and C. S. Bok. "Optimal design of a T-shaped drum-type brake for motorcycle utilizing magnetorheological fluid," Mech. Based Des. Struct. Mach., vol/issue: 40(2), pp. 153-162, 2012.

[12] T. M Avraam, "MR-fluid brake design and its application to a portable muscular rehabilitation device," Universit' e Libre de Bruxelles F., 2009.

[13] Ubaidillah, et al., "Performance prediction of serpentine type compact magnetorheological brake prototype," AIP Conf. Proc., pp. 1788, 2017.

[14] Y. Shiao and Q. A. Nguyen, "Torque enhancement for a new magnetorheological brake," Procedia Eng., vol/issue: 76(1), pp. 12-23, 2014.

[15] Ossurs Inc. USD446304, 2011.

[16] M. Avraam, et al., "Computer Controlled Rotational MR-brake for Wrist Rehabilitation Device," J. Intell. Mater. Syst. Struct., vol/issue: 21(15), pp. 1543-1557, 2011.

[17] K. H. Gudmundsson, et al., "A geometrical optimization of a magneto-rheological rotary brake in a prosthetic knee,” Smart Mater. Struct., vol/issue: 19(3), pp. 35023, 2010.

[18] J. D. Carlson, United States Patent 191, 1998.

[19] D. Senkal and H. Gurocak, "Serpentine flux path for high torque MRF brakes in haptics applications," Mechatronics, vol/issue: 20(3), pp. 377-383, 2010.

\section{BIOGRAPHIES OF AUTHORS}

Faishal harish hidayatullah received his bachelor degree from Mechanical Engineering, Faculty
of Engineering, Universitas Sebelas Maret in 2018. He is currently management trainee in
PAMA PERSADA. His fonal project research is about design and development of
Magnetorheological Brake for foot ankle orthosis.

